\title{
Properties of $q$-shift difference-differential polynomials of meromorphic functions
}

\author{
Xin-Li Wang ${ }^{1}$, Hong-Yan Xu ${ }^{2 *}$ and Tang-Sen Zhan ${ }^{2}$
}

\section{"Correspondence:}

xhyhhh@126.com

2Department of Informatics and

Engineering, Jingdezhen Ceramic

Institute, Jingdezhen, Jiangxi

333403, China

Full list of author information is

available at the end of the article

\begin{abstract}
In this paper, we deal with the zeros of the $q$-shift difference-differential polynomials $\left[P(f) \prod_{j=1}^{d} f\left(q_{j} z+c_{j}\right)^{s_{j}}\right]^{(k)}-\alpha(z)$ and $\left(P(f) \prod_{j=1}^{d}\left[f\left(q_{j} z+c_{j}\right)-f(z)\right]^{s_{j}}\right)^{(k)}-\alpha(z)$, where $P(f)$ is a nonzero polynomial of degree $n, q_{j}, c_{j} \in \mathbb{C} \backslash\{0\}(j=1, \ldots, d)$ are constants, $n, d, s_{j}(j=1, \ldots, d) \in \mathbb{N}_{+}$and $\alpha(z)$ is a small function of $f$. The results of this paper are an extension of the previous theorems given by Chen and Chen and Qi. We also investigate the value sharing for $q$-shift difference polynomials of entire functions and obtain some results which extend the recent theorem given by Liu, Liu and Cao.
\end{abstract}

MSC: 39A50; 30D35

Keywords: $q$-shift; uniqueness; meromorphic function; zero order

\section{Introduction and main results}

The purpose of this paper is to study some properties of zeros and uniqueness of complex $q$-shift difference polynomials of meromorphic functions. A polynomial $Q_{q}(z, f)$ can be called a $q$-shift difference-differential polynomial in $f$ whenever $Q_{q}(z, f)$ is a polynomial in $f(z)$, its $q$-shift $f(q z+c)$ and their derivatives, with small functions of $f(z)$ as the coefficients. The fundamental results and the standard notations of the Nevanlinna value distribution theory of meromorphic functions will be used (see [1,2]). A meromorphic function $f$ means $f$ is meromorphic in the complex plane. If no poles occur, then $f$ reduces to an entire function. Given a meromorphic function $f(z)$, a meromorphic function $a(z)$ is called a small function with respect to $f$ if $T(r, a(z))=S(r, f)$, where $S(r, f)$ is used to denote any quantity satisfying $S(r, f)=o(T(r, f))$ for all $r$ outside a possible exceptional set $E$ of finite logarithmic measure $\lim _{r \rightarrow \infty} \int_{[1, r) \cap E} \frac{d t}{t}<\infty$. We also use $S_{1}(r, f)$ to denote any quantity satisfying $S_{1}(r, f)=o(T(r, f))$ for all $r$ on a set $F$ of logarithmic density 1 ; the logarithmic density of a set $F$ is defined by

$$
\limsup _{r \rightarrow \infty} \frac{1}{\log r} \int_{[1, r] \cap F} \frac{1}{t} d t
$$

In addition, for some $a \in \mathbb{C} \cup\{\infty\}$, if the zeros of $f(z)-a$ and $g(z)-a$ (if $a=\infty$, zeros of $f(z)-a$ and $g(z)-a$ are the poles of $f(z)$ and $g(z)$, respectively) coincide in locations and multiplicities we say that $f(z)$ and $g(z)$ share the value $a C M$ (counting multiplicities) and if they coincide in locations only we say that $f(z)$ and $g(z)$ share $a I M$ (ignoring multiplicities).

@ 2014 Wang et al.; licensee Springer. This is an Open Access article distributed under the terms of the Creative Commons Attribution License (http://creativecommons.org/licenses/by/2.0), which permits unrestricted use, distribution, and reproduction in any medium, provided the original work is properly cited. 
Definition 1.1 (see $[3,4]$ ) Let $l$ be a nonnegative integer or infinity. For $a \in \mathbb{C} \cup\{\infty\}$, we denote by $E_{l}(a ; f)$ the set of all $a$-points of $f$ where an $a$-point of multiplicity $k$ is counted $k$ times if $k \leq l$ and $l+1$ times if $k>l$. If $E_{l}(a ; f)=E_{l}(a ; g)$, we say that $f, g$ share the value $a$ with weight $l$.

Definition 1.2 (see [5]) When $f$ and $g$ share $1 I M$, we denote by $\bar{N}_{L}\left(r, \frac{1}{f-1}\right)$ the counting function of the 1-points of $f$ whose multiplicities are greater than 1-points of $g$, where each zero is counted only once; similarly, we have $\bar{N}_{L}\left(r, \frac{1}{g-1}\right)$. Let $z_{0}$ be a zero of $f-1$ of multiplicity $p$ and a zero of $g-1$ of multiplicity $q$, we also denote by $N_{11}\left(r, \frac{1}{f-1}\right)$ the counting function of those 1-points of $f$ where $p=q=1$.

In recent years, there has been an increasing interest in studying difference equations, difference products and $q$-differences in the complex plane $\mathbb{C}$, and a number of papers (including [6-13]) have focused on the value distribution and uniqueness of differences and differences operator analogs of Nevanlinna theory.

For a transcendental meromorphic function $f$ of finite order, herein and hereinafter, $c$ is a nonzero complex constant and $a(z)$ is small function with respect to $f$, Liu et al. [14], Chen et al. [15], and Luo and Lin [16] studied the zeros distributions of difference polynomials of meromorphic functions and obtained: if $n \geq 6$, then $f(z)^{n} f(z+c)-\alpha(z)$ has infinitely many zeros [14, Theorem 1.2]; if $n>m$, then $P(f) f(z+c)-a(z)$ has infinitely many zeros (see [16, Theorem 1]), where $P(z)=a_{n} z^{n}+a_{n-1} z^{n-1}+\cdots+a_{1} z+a_{0}$ is a nonzero polynomial, where $a_{0}, a_{1}, \ldots, a_{n}(\neq 0)$ are complex constants, and $m$ is the number of the distinct zeros of $P(z)$.

For transcendental meromorphic (resp. entire) function $f$ of zero order and nonzero complex constant $q$, Zhang and Korhonen [17] studied the value distribution of $q$ difference polynomials of meromorphic functions and obtained the result that if $n \geq 6$ (resp. $n \geq 2$ ), then $f(z)^{n} f(q z)$ assumes every nonzero value $a \in \mathbb{C}$ infinitely often (see [17, Theorem 4.1]).

Recently, Liu and Qi [18] firstly investigated the value distributions for $q$-shift of meromorphic function and obtained the following result.

Theorem A (see [18, Theorem 3.6]) Let $f$ be a zero-order transcendental meromorphic function, $n \geq 6, q \in \mathbb{C} \backslash\{0\}, \eta \in \mathbb{C}$, and let $R(z)$ be a rational function. Then the $q$-shift difference polynomial $f(z)^{n} f(q z+\eta)-R(z)$ has infinitely many zeros.

In this paper, we assume $q_{j}, c_{j} \in \mathbb{C} \backslash\{0\}(j=1, \ldots, d)$ are constants, $n, d, s_{j}(j=1, \ldots, d) \in$ $\mathbb{N}_{+}, \lambda=s_{1}+s_{2}+\cdots+s_{d}$, and $a(z)$ is a small function of $f$. We will study the value distribution of difference polynomials of more general form,

$$
\begin{aligned}
& F(z)=P(f) \prod_{j=1}^{d} f\left(q_{j} z+c_{j}\right)^{s_{j}}, \\
& F_{1}(z)=P(f) \prod_{j=1}^{d}\left[f\left(q_{j} z+c_{j}\right)-f(z)\right]^{s_{j}},
\end{aligned}
$$

where $P(f)$ is a nonzero polynomial of degree $n, m$ is the number of the distinct zeros of $P(z)$, and we obtain the following results. 
Theorem 1.1 Let $f$ be a transcendental meromorphic (resp. entire) function of zero order and $F(z)$ be stated as in (1). If $k \in \mathbb{N}$ and $n>m(k+1)+2 d+1+\lambda($ resp. $n>m(k+1)+d-\lambda)$. Then $(F(z))^{(k)}-a(z)$ has infinitely many zeros, where $(F(z))^{(k)}=F(z)$, if $k=0$.

Theorem 1.2 Let $f$ be a transcendental meromorphic (resp. entire) function of zero order and $F_{1}(z)$ be stated as in (2). Assume $k \in \mathbb{N}$ and $n>(m+2 d)(k+1)+\lambda+d+1($ resp. $n>(m+$ $2 d)(k+1))$. Then $\left(F_{1}(z)\right)^{(k)}-a(z)$ has infinitely many zeros, provided that $f\left(q_{j} z+c_{j}\right) \neq f(z)$, $j=1,2, \ldots, d$.

Recently, there were obtained some results on the existence and growth of solutions of difference-differential equations (see $[19,20]$ ). Here, from Theorem 1.1 and Theorem 1.2, we get the following result on some nonlinear $q$-shift difference-differential equations.

Corollary 1.1 Let $p(z), q(z)$ be nonzero polynomials and $F(z)$ be stated as in (1). Then the nonlinear q-shift difference-differential equation

$$
[F(z)]^{(k)}-p(z)=q(z)
$$

has no transcendental meromorphic solution of zero order, provided that $n \geq \lambda+1$.

Corollary 1.2 Let $p(z), q(z)$ be nonzero polynomials and $F_{1}(z)$ be stated as in (2). Then the nonlinear $q$-shift difference-differential equation

$$
\left[F_{1}(z)\right]^{(k)}-p(z)=q(z)
$$

has no transcendental meromorphic solution of zero order, provided that $f\left(q_{j} z+c_{j}\right) \neq f(z)$, $j=1,2, \ldots, d$, and $n \geq \lambda+1$.

For the uniqueness of difference and $q$-difference of meromorphic functions, some results had been obtained (see [17, 21-23]). Here, we only state some of the latest theorems as follows.

Theorem B (see [16, Theorem 2]) Let $f$ and $g$ be transcendental entire functions of finite order, c be a nonzero complex constant, $P(z)$ be stated as in Theorem 1.3 , and let $n>2 \Gamma_{0}+1$ be an integer, where $\Gamma_{0}=m_{1}+2 m_{2}, m_{1}$ is the number of the simple zero of $P(z)$, and $m_{2}$ is the number of multiple zeros of $P(z)$. If $P(f) f(z+c)$ and $P(g) g(z+c)$ share $1 C M$, then one of the following results holds:

(i) $f \equiv$ tg for a constant $t$ such that $t^{l}=1$, where $l=G C D\left\{\lambda_{0}, \lambda_{1}, \ldots, \lambda_{n}\right\}$ and

$$
\lambda_{i}=\left\{\begin{array}{ll}
i+1, & a_{i} \neq 0, \\
n+1, & a_{i}=0,
\end{array} \quad i=0,1,2, \ldots, n\right.
$$

(ii) $f$ and $g$ satisfy the algebraic equation $R(f, g) \equiv 0$, where

$$
R\left(\omega_{1}, \omega_{2}\right)=P\left(\omega_{1}\right) \omega_{1}(z+c)-P\left(\omega_{2}\right) \omega_{2}(z+c) ;
$$

(iii) $f(z)=e^{\alpha(z)}, g(z)=e^{\beta(z)}$, where $\alpha(z)$ and $\beta(z)$ are two polynomials, $b$ is a constant satisfying $\alpha+\beta \equiv b$ and $a_{n}^{2} e^{(n+1) b}=1$. 
In this paper, we will investigated the uniqueness problem of $q$-shifts of entire functions and obtain the following results.

Theorem 1.3 Let $f$, $g$ be transcendental entire functions of zero order, $F(z)$ be stated as in (1) and

$$
G(z)=P(g) \prod_{j=1}^{d} g\left(q_{j} z+c_{j}\right)^{s_{j}}
$$

where $\Gamma_{0}$ is stated as in Theorem B. If $F(z)$ and $G(z)$ share $1 C M$ and $n>\max \left\{2\left(\Gamma_{0}+2 d\right)-\right.$ $\lambda, \lambda\}$, then one of the following cases holds:

(i) $f \equiv$ tg for a constant $t$ such that $t^{\kappa}=1$ where $\kappa=G C D\left\{\lambda_{0}+\lambda, \lambda_{1}+\lambda, \ldots, \lambda_{n}+\lambda\right\}$ and

$$
\lambda_{i}=\left\{\begin{array}{ll}
i+1, & a_{i} \neq 0, \\
n+1, & a_{i}=0,
\end{array} \quad i=0,1,2, \ldots, n\right.
$$

(ii) $f$ and $g$ satisfy the algebraic equation $R(f, g) \equiv 0$, where

$$
R\left(\omega_{1}, \omega_{2}\right)=P\left(\omega_{1}\right) \prod_{j=1}^{d} \omega_{1}\left(q_{j} z+c_{j}\right)^{s_{j}}-P\left(\omega_{2}\right) \prod_{j=1}^{d} \omega_{2}\left(q_{j} z+c_{j}\right)^{s_{j}}
$$

Theorem 1.4 Under the assumptions of Theorem 1.3, if

$$
E_{l}\left(1 ; P(f) \prod_{j=1}^{d} f\left(q_{j} z+c_{j}\right)^{s_{j}}\right)=E_{l}\left(1 ; P(g) \prod_{j=1}^{d} g\left(q_{j} z+c_{j}\right)^{s_{j}}\right)
$$

and $l, n, m$ are integers satisfying one of the following conditions:

(I) $l \geq 3, n>\max \left\{2 \Gamma_{0}+4 d-\lambda, \lambda\right\}$;

(II) $l=2, n>\max \left\{2 \Gamma_{0}+5 d+m-\lambda-d \chi, \lambda\right\}$;

(III) $l=1, n>\max \left\{2 \Gamma_{0}+6 d+2 m-\lambda-2 d \chi, \lambda\right\}$;

(IV) $l=0, n>\max \left\{2 \Gamma_{0}+7 d+3 m-\lambda-3 d \chi, \lambda\right\}$.

Then the conclusions of Theorem 1.3 hold, where $\chi=\min \{\Theta(0, f), \Theta(0, g)\}$.

\section{Some lemmas}

In the following, we explain some definitions and notations which are used in this paper. For $a \in \mathbb{C} \cup\{\infty\}$, we define

$$
\Theta(a, f)=1-\limsup _{r \rightarrow \infty} \frac{\bar{N}\left(r, \frac{1}{f-a}\right)}{T(r, f)} .
$$

For $a \in \mathbb{C} \cup\{\infty\}$ and $k$ is a positive integer, we denote by $\bar{N}_{(k}\left(r, \frac{1}{f-a}\right)$ the counting function of those $a$-points of $f$ whose multiplicities are not less than $k$ in counting the $a$-points of $f$ we ignore the multiplicities (see [1]) and $N_{k}\left(r, \frac{1}{f-a}\right)=\bar{N}\left(r, \frac{1}{f-a}\right)+\bar{N}_{(2}\left(r, \frac{1}{f-a}\right)+\cdots+$ $\bar{N}_{(k}\left(r, \frac{1}{f-a}\right)$.

Lemma 2.1 (see [2]) Let $f$ and $g$ be two meromorphic functions. Iff and $g$ share 1 CM, then one of the following three cases holds: 
(i) $T(r, f)+T(r, g) \leq 2 N_{2}(r, f)+2 N_{2}(r, g)+2 N_{2}\left(r, \frac{1}{f}\right)+2 N_{2}\left(r, \frac{1}{g}\right)+S(r, f)+S(r, g)$;

(ii) $f \equiv g$;

(iii) $f \cdot g=1$.

Lemma 2.2 (see [24]) Let $f$ and $g$ be two meromorphic functions, and let $l$ be a positive integer. If $E_{l}(1 ; f)=E_{l}(1 ; g)$, then one of the following cases must occur:

(i)

$$
\begin{aligned}
T(r, f)+T(r, g) \leq & N_{2}(r, f)+N_{2}(r, g)+N_{2}\left(r, \frac{1}{f}\right)+N_{2}\left(r, \frac{1}{g}\right)+\bar{N}\left(r, \frac{1}{f-1}\right) \\
& +\bar{N}\left(r, \frac{1}{g-1}\right)-N_{11}\left(r, \frac{1}{f-1}\right)+\bar{N}_{(l+1}\left(r, \frac{1}{f-1}\right) \\
& +\bar{N}_{(l+1}\left(r, \frac{1}{g-1}\right)+S(r, f)+S(r, g) ;
\end{aligned}
$$

(ii) $f=\frac{(b+1) g+(a-b-1)}{b g+(a-b)}$, where $a(\neq 0), b$ are two constants.

Lemma 2.3 (see [24]) Let $f$ and $g$ be two meromorphic functions. If $f$ and $g$ share 1 IM, then one of the following cases must occur:

(i)

$$
\begin{aligned}
T(r, f)+T(r, g) \leq & 2\left[N_{2}(r, f)+N_{2}\left(r, \frac{1}{f}\right)+N_{2}(r, g)+N_{2}\left(r, \frac{1}{g}\right)\right] \\
& +3 \bar{N}_{L}\left(r, \frac{1}{f-1}\right)+3 \bar{N}_{L}\left(r, \frac{1}{g-1}\right)+S(r, f)+S(r, g) ;
\end{aligned}
$$

(ii) $f=\frac{(b+1) g+(a-b-1)}{b g+(a-b)}$, where $a(\neq 0), b$ are two constants.

By combining [7] and [17], we get the following lemma easily.

Lemma 2.4 Let $f(z)$ be a transcendental meromorphic function of zero order and $q, \eta$ be two nonzero complex constants. Then

$$
\begin{aligned}
& T(r, f(q z+\eta))=T(r, f(z))+S_{1}(r, f), N\left(r, \frac{1}{f(q z+\eta)}\right)=N\left(r, \frac{1}{f}\right)+S_{1}(r, f), \\
& N(r, f(q z+\eta))=N(r, f)+S_{1}(r, f), \bar{N}\left(r, \frac{1}{f(q z+\eta)}\right)=\bar{N}\left(r, \frac{1}{f}\right)+S_{1}(r, f), \\
& \bar{N}(r, f(q z+\eta))=\bar{N}(r, f)+S_{1}(r, f) .
\end{aligned}
$$

Lemma 2.5 (see [18, Theorem 2.1]) Let $f(z)$ be a nonconstant zero-order meromorphic function and $q \in \mathbb{C} \backslash\{0\}$. Then

$$
m\left(r, \frac{f(q z+\eta)}{f(z)}\right)=S(r, f)
$$

on a set of logarithmic density 1. 
Lemma 2.6 Let $f$ be a transcendental meromorphic function of zero order, and $F(z)$ be stated as in (1). Then we have

$$
(n-\lambda) T(r, f)+S_{1}(r, f) \leq T(r, F(z)) \leq(n+\lambda) T(r, f)+S_{1}(r, f) .
$$

Iff is a transcendental entire function of zero order, we have

$$
T(r, F(z))=T\left(r, P(f) f^{\lambda}\right)+S_{1}(r, f)=(n+\lambda) T(r, f)+S_{1}(r, f),
$$

where $\lambda=s_{1}+s_{2}+\cdots+s_{d}$.

Proof If $f$ is a transcendental entire function of zero order, from the Valiron-Mohon'ko lemma and Lemma 2.5 , we have

$$
\begin{aligned}
T(r, F(z)) & =m(r, F(z)) \leq m\left(r, P(f) f^{\lambda}(z)\right)+m\left(r, \frac{\prod_{j=1}^{d} f\left(q_{j} z+c_{j}\right)^{s_{j}}}{f^{\lambda}(z)}\right) \\
& \leq m\left(r, P(f) f^{\lambda}(z)\right)+S_{1}(r, f)=T\left(r, P(f) f^{\lambda}(z)\right)+S_{1}(r, f) \\
& =(n+\lambda) T(r, f)+S_{1}(r, f) .
\end{aligned}
$$

On the other hand, from Lemma 2.5, we have

$$
\begin{aligned}
(n+\lambda) T(r, f) & =T\left(r, P(f) f^{\lambda}(z)\right)+S(r, f)=m\left(r, P(f) f^{\lambda}(z)\right)+S(r, f) \\
& \leq m(r, F(z))+m\left(r, \frac{f^{\lambda}(z)}{\prod_{j=1}^{d} f\left(q_{j} z+c_{j}\right)^{s_{j}}}\right) \\
& =T(r, F(z))+S_{1}(r, f) .
\end{aligned}
$$

Thus, we get (6).

If $f$ is a meromorphic function of zero order, from the Valiron-Mohon'ko lemma and Lemma 2.4, we have

$$
T\left(r, P(f) \prod_{j=1}^{d} f\left(q_{j} z+c_{j}\right)^{s_{j}}\right) \leq T(r, P(f))+T\left(r, \prod_{j=1}^{d} f\left(q_{j} z+c_{j}\right)^{s_{j}}\right) \leq(n+\lambda) T(r, f)+S_{1}(r, f) .
$$

On the other hand, from the Valiron-Mo'honko lemma and Lemma 2.5, we have

$$
\begin{aligned}
(n+\lambda) T(r, f)= & T\left(r, P(f) f^{\lambda}\right)+S(r, f)=m\left(r, P(f) f^{\lambda}\right)+N\left(r, P(f) f^{\lambda}\right)+S(r, f) \\
\leq & m\left(r, F(z) \frac{f^{\lambda}(z)}{\prod_{j=1}^{d} f\left(q_{j} z+c_{j}\right)^{s_{j}}}\right) \\
& +N\left(r, F(z) \frac{f^{\lambda}(z)}{\prod_{j=1}^{d} f\left(q_{j} z+c_{j}\right)^{s_{j}}}\right)+S(r, f) \\
\leq & T(r, F(z))+2 \lambda T(r, f)+S_{1}(r, f) .
\end{aligned}
$$

Thus, we get (5).

Using the same method as in Lemma 2.6, we get the following lemma easily. 
Lemma 2.7 Let $f$ be a transcendental meromorphic function of zero order, and $F_{1}(z)$ be stated as in (2). Then we have

$$
(n-\lambda) T(r, f)+S_{1}(r, f) \leq T\left(r, F_{1}(z)\right) \leq(n+2 \lambda) T(r, f)+S_{1}(r, f) .
$$

Iff is a transcendental entire function of zero order, we have

$$
T\left(r, F_{1}(z)\right)=T\left(r, P(f) \prod_{j=1}^{d}\left[f\left(q_{j} z+c_{j}\right)-f(z)\right]^{s_{j}}\right) \geq n T(r, f)+S_{1}(r, f) .
$$

Lemma 2.8 (see [2] and [25, Lemma 2.4]) Letf be a nonconstant meromorphic function, and $p, k$ be positive integers. Then

$$
\begin{aligned}
& T\left(r, f^{(k)}\right) \leq T(r, f)+k \bar{N}(r, f)+S(r, f) \\
& N_{p}\left(r, \frac{1}{f^{(k)}}\right) \leq T\left(r, f^{(k)}\right)-T(r, f)+N_{p+k}\left(r, \frac{1}{f}\right)+S(r, f), \\
& N_{p}\left(r, \frac{1}{f^{(k)}}\right) \leq k \bar{N}(r, f)+N_{p+k}\left(r, \frac{1}{f}\right)+S(r, f) .
\end{aligned}
$$

Lemma 2.9 Let $f(z)$ and $g(z)$ be transcendental entire functions of zero order, $P(z)$ be stated as in Theorem 1.1. If $n>\lambda$, then for any complex constant $t \neq 0$, we have

$$
P(f) \prod_{j=1}^{d} f\left(q_{j} z+c_{j}\right)^{s_{j}} P(g) \prod_{j=1}^{d} g\left(q_{j} z+c_{j}\right)^{s_{j}} \not \equiv t .
$$

Proof For any complex constant $t \neq 0$, suppose that

$$
P(f) \prod_{j=1}^{d} f\left(q_{j} z+c_{j}\right)^{s_{j}} P(g) \prod_{j=1}^{d} g\left(q_{j} z+c_{j}\right)^{s_{j}} \equiv t .
$$

Suppose that the roots of $P(z)=0$ are $b_{1}, b_{2}, \ldots, b_{m}$ with multiplicities $l_{1}, l_{2}, \ldots, l_{m}$. Then we have $l_{1}+l_{2}+\cdots+l_{m}=n$. From (7), we have

$$
\begin{aligned}
& \left(f-b_{1}\right)^{l_{1}}\left(f-b_{2}\right)^{l_{2}} \cdots\left(f-b_{m}\right)^{l_{m}} \prod_{j=1}^{d} f\left(q_{j} z+c_{j}\right)^{s_{j}} \\
& \quad \times\left(g-b_{1}\right)^{l_{1}}\left(g-b_{2}\right)^{l_{2}} \cdots\left(g-b_{m}\right)^{l_{m}} \prod_{j=1}^{d} g\left(q_{j} z+c_{j}\right)^{s_{j}} \equiv t .
\end{aligned}
$$

Since $f, g$ are nonconstant entire functions, from (8), we deduce that $b_{1}=b_{2}=\cdots=b_{m}=0$. If fact, from (8), we get that $b_{1}, b_{2}, \ldots, b_{m}$ are the Picard exceptional values. If $m \geq 2$ and $b_{j} \neq 0(j=1,2, \ldots, m)$, by Picard's theorem of entire function, we can see that the Picard exceptional values of $f$ are at least three. Thus, we get a contradiction. Hence, $m=1$ and $l_{1}=n$, that is, there exists a complex constant $\gamma$ satisfying $P(f)=a_{n}(f-\gamma)^{n}$ and $P(g)=$ 
$a_{n}(g-\gamma)^{n}$. Then

$$
a_{n}(f-\gamma)^{n} \prod_{j=1}^{d} f\left(q_{j} z+c_{j}\right)^{s_{j}} a_{n}(g-\gamma)^{n} \prod_{j=1}^{d} g\left(q_{j} z+c_{j}\right)^{s_{j}} \equiv t .
$$

Since $f, g$ are transcendental entire functions, by the Picard theorem, we can see that $f$ $\gamma=0$ and $g-\gamma=0$ do not have zeros. Then we obtain $f(z)=e^{\alpha(z)}+\gamma, g(z)=e^{\beta(z)}+\gamma$ where $\alpha(z), \beta(z)$ are two nonconstant functions. From (9), we see that $f\left(q_{j} z+c_{j}\right) \neq 0$ and $g\left(q_{j} z+c_{j}\right) \neq 0$. Thus, we get $\gamma=0$, that is,

$$
a_{n}^{2} f(z)^{n} \prod_{j=1}^{d} f\left(q_{j} z+c_{j}\right)^{s_{j}} g(z)^{n} \prod_{j=1}^{d} g\left(q_{j} z+c_{j}\right)^{s_{j}} \equiv t .
$$

Set $M(z)=f(z) g(z)$. If $M(z)$ is nonconstant, from (10), we have

$$
a_{n}^{2} M(z)^{n} \prod_{j=1}^{d} M\left(q_{j} z+c_{j}\right)^{s_{j}} \equiv t
$$

that is,

$$
a_{n}^{2} M(z)^{n} \equiv \frac{t}{\prod_{j=1}^{d} M\left(q_{j} z+c_{j}\right)^{s_{j}}} .
$$

Since $f, g$ are transcendental entire functions of zero order, from (11), Lemma 2.4 and $n>\lambda$, we get a contradiction.

Thus, $M(z)$ is a constant. From (11), we get $f(z) g(z) \equiv \mu$, where $\mu$ is a complex constant satisfying $a_{n}^{2} \mu^{n+\lambda} \equiv t$. Since $f, g$ are entire functions of zero order, then $f, g$ are constants, which is a contradiction with $f, g$ being transcendental. Hence,

$$
P(f) \prod_{j=1}^{d} f\left(q_{j} z+c_{j}\right)^{s_{j}} P(g) \prod_{j=1}^{d} g\left(q_{j} z+c_{j}\right)^{s_{j}} \not \equiv t .
$$

This completes the proof of Lemma 2.9.

\section{Proofs of Theorems 1.1 and 1.2}

Proof of Theorem 1.1 From (1), by the Valiron-Mohon'ko lemma and Lemma 2.6, we find that $F(z)$ is not constant and $S_{1}\left(r, F^{(k)}\right)=S_{1}(r, F)=S_{1}(r, f)$. Next, we will consider the following two cases when $k \geq 1$.

Case 1. If $f$ is a transcendental meromorphic function of zero order, we first suppose that $\left(P(f) \prod_{j=1}^{d} f\left(q_{j} z+c_{j}\right)^{s_{j}}\right)^{(k)}=a(z)$ has finitely solutions. By the Second Fundamental Theorem for three small functions (see [1, Theorem 2.25]) and the Valiron-Mohon'ko lemma, we 
have

$$
\begin{aligned}
T\left(r, F^{(k)}\right) & \leq \bar{N}\left(r, F^{(k)}\right)+\bar{N}\left(r, \frac{1}{F^{(k)}}\right)+\bar{N}\left(r, \frac{1}{F^{(k)}-a(z)}\right)+S\left(r, F^{(k)}\right) \\
& \leq \bar{N}(r, f)+\sum_{j=1}^{d} \bar{N}\left(r, f\left(q_{j} z+c_{j}\right)\right)+N_{1}\left(r, \frac{1}{F^{(k)}}\right)+S\left(r, F^{(k)}\right) \\
& \leq(d+1) T(r, f)+T\left(r, F^{(k)}\right)-T(r, F)+N_{k+1}\left(r, \frac{1}{F^{(k)}}\right)+S\left(r, F^{(k)}\right) .
\end{aligned}
$$

By Lemma 2.6 and Lemma 2.8, we obtain

$$
\begin{aligned}
(n- & \lambda) T(r, f)+S_{1}(r, f) \\
& \leq T(r, F) \leq(d+1) T(r, f)+N_{k+1}\left(r, \frac{1}{F^{(k)}}\right)+S(r, f) \\
& \leq(d+1) T(r, f)+m(k+1) \bar{N}\left(r, \frac{1}{f}\right)+\sum_{j=1}^{d} N\left(r, \frac{1}{f\left(q_{j} z+c_{j}\right)}\right)+S_{1}(r, f)+S(r, f) \\
& \leq[m(k+1)+2 d+1] T(r, f)+S_{1}(r, f)+S(r, f) .
\end{aligned}
$$

From the definitions of $S_{1}(r, f), S(r, f)$ and $n>m(k+1)+2 d+1+\lambda$, we get a contradiction to (12). Then $F(z)^{(k)}-a(z)$ has infinitely many zeros.

Case 2. If $f$ is a transcendental entire function. Suppose that $\left(P(f) \prod_{j=1}^{d} f\left(q_{j} z+c_{j}\right)^{s_{j}}\right)^{(k)}=$ $a(z)$ has finitely solutions. By using the same argument as in Case 1 and (4), we have

$$
(n+\lambda) T(r, f)+S_{1}(r, f) \leq[m(k+1)+d] T(r, f)+S_{1}(r, f)+S(r, f),
$$

which is a contradiction with $n>m(k+1)+d-\lambda$.

For $k=0$, similar to the proofs of Case 1 and Case 2, and by the Second Fundamental Theorem and Lemma 2.6, we get the conclusions of Theorem 1.1.

Thus, we complete the proof of Theorem 1.1.

Proof of Theorem 1.2 Similar to the proof of Theorem 1.1, and using Lemma 2.8, we can prove Theorem 1.2 easily.

\section{Proofs of Corollaries $\mathbf{1 . 1}$ and $\mathbf{1 . 2}$}

The proofs of Corollaries 1.1 and 1.2 are similar. Here, we just give the proof of Corollary 1.2 .

Proof of Corollary 1.2 Assume that $f$ is a transcendental meromorphic solution of zero order of (4), provided that $f\left(q_{j} z+c_{j}\right) \neq f(z), j=1,2, \ldots, d$, then

$$
\left[P(f) \prod_{j=1}^{d}\left[f\left(q_{j} z+c_{j}\right)-f(z)\right]^{s_{j}}\right]^{(k)}=p(z)+q(z) .
$$


Integrating above equation $k$ times, it follows

$$
\frac{H(z)}{P(f) f^{\lambda}}=\frac{\prod_{j=1}^{d}\left[f\left(q_{j} z+c_{j}\right)-f(z)\right]^{s_{j}}}{f^{\lambda}}
$$

where $H(z)$ is a polynomial. From Lemma 2.5 and since $f$ is a meromorphic function of zero order, we have

$$
\begin{aligned}
(n+\lambda) T(r, f) & =T\left(r, \frac{\prod_{j=1}^{d}\left[f\left(q_{j} z+c_{j}\right)-f(z)\right]^{s_{j}}}{f^{\lambda}}\right) \\
& =N\left(r, \frac{\prod_{j=1}^{d}\left[f\left(q_{j} z+c_{j}\right)-f(z)\right]^{s_{j}}}{f^{\lambda}}\right)+S_{1}(r, f) \\
& \leq 2 \lambda T(r, f)+S_{1}(r, f),
\end{aligned}
$$

which is contradiction with $n>\lambda$.

This completes the proof of Corollary 1.2.

\section{Proofs of Theorems 1.3 and 1.4}

Proof of Theorem 1.3 From the assumptions of Theorem 1.3, we see that $F(z), G(z)$ share $1 C M$. Then the following three cases will be considered.

Case 1. Suppose that $F(z), G(z)$ satisfy Lemma 2.1(i). Since $f(z), g(z)$ are entire functions of zero order, from the Valiron-Mohon'ko lemma and Lemma 2.6, we have $S_{1}(r, F)=$ $S_{1}(r, f), S_{1}(r, G)=S_{1}(r, g)$,

$$
\begin{aligned}
N_{2}\left(r, \frac{1}{F}\right) & \leq N_{2}\left(r, \frac{1}{P(f)}\right)+\sum_{j=1}^{d} N_{2}\left(r, \frac{1}{f\left(q_{j} z+c_{j}\right)^{s_{j}}}\right) \\
& \leq \Gamma_{0} T(r, f)+2 \sum_{j=1}^{d} \bar{N}\left(r, \frac{1}{f\left(q_{j} z+c_{j}\right)}\right) \leq\left(\Gamma_{0}+2 d\right) T(r, f)+S_{1}(r, f),
\end{aligned}
$$

and

$$
N_{2}\left(r, \frac{1}{G}\right) \leq\left(\Gamma_{0}+2 d\right) T(r, g)+S_{1}(r, g) .
$$

Then, from Lemma 2.1(i) and Lemma 2.7, since $f, g$ are entire, we have

$$
\begin{aligned}
T(r, F(z))+T(r, G(z)) & \leq 2 N_{2}\left(r, \frac{1}{F}\right)+2 N_{2}\left(r, \frac{1}{G}\right)+S(r, f)+S(r, g) \\
& \leq 2\left(\Gamma_{0}+2 d\right)[T(r, f)+T(r, g)]+S_{1}(r, f)+S_{1}(r, g) .
\end{aligned}
$$

From Lemma 2.6 and (12), we have

$$
(n+\lambda)[T(r, f)+T(r, g)] \leq 2\left(\Gamma_{0}+2 d\right)[T(r, f)+T(r, g)]+S_{1}(r, f)+S_{1}(r, g),
$$


that is,

$$
\left(n+\lambda-2 \Gamma_{0}-4 d\right)[T(r, f)+T(r, g)] \leq S_{1}(r, f)+S_{1}(r, g) .
$$

Since $n>2\left(\Gamma_{0}+2 d\right)-\lambda$ and $f, g$ are transcendental functions, we get a contradiction.

Case 2. If $F(z) \equiv G(z)$, that is,

$$
P(f) \prod_{j=1}^{d} f\left(q_{j} z+c_{j}\right)^{s_{j}} \equiv P(g) \prod_{j=1}^{d} g\left(q_{j} z+c_{j}\right)^{s_{j}} .
$$

Set $h=\frac{f}{g}$. If $h$ is not a constant, from (17), we find that $f$ and $g$ satisfy the algebraic equation $R(f, g) \equiv 0$, where

$$
R\left(\omega_{1}, \omega_{2}\right)=P\left(\omega_{1}\right) \prod_{j=1}^{d} \omega_{1}\left(q_{j} z+c_{j}\right)^{s_{j}}-P\left(\omega_{2}\right) \prod_{j=1}^{d} \omega_{2}\left(q_{j} z+c_{j}\right)^{s_{j}} .
$$

If $h$ is a constant. Substituting $f=g h$ into (17), we get

$$
\prod_{j=1}^{d} g\left(q_{j} z+c_{j}\right)^{s_{j}}\left[a_{n} g^{n}\left(h^{n+\lambda}-1\right)+a_{n-1} g^{n-1}\left(h^{n+\lambda-1}-1\right)+\cdots+a_{0}\left(h^{\lambda}-1\right)\right] \equiv 0,
$$

where $a_{n}(\neq 0), a_{n-1}, \ldots, a_{0}$ are constants.

Since $g$ is transcendental entire function, we have $\prod_{j=1}^{d} g\left(q_{j} z+c_{j}\right)^{s_{j}} \not \equiv 0$. Then, from (18), we have

$$
a_{n} g^{n}\left(h^{n+\lambda}-1\right)+a_{n-1} g^{n-1}\left(h^{n+\lambda-1}-1\right)+\cdots+a_{0}\left(h^{\lambda}-1\right) \equiv 0 .
$$

If $a_{n} \neq 0$ and $a_{n-1}=a_{n-2}=\cdots=a_{0}=0$, then from (19) and $g$ is transcendental function, we get $h^{n+\lambda}=1$.

If $a_{n} \neq 0$ and there exists $a_{i} \neq 0(i \in\{0,1,2, \ldots, n-1\})$. Suppose that $h^{n+\lambda} \neq 1$, from (19), we have $T(r, g)=S(r, g)$ which is contradiction with transcendental function $g$. Then $h^{n+\lambda}=1$. Similar to this discussion, we can see that $h^{j+\lambda}=1$ when $a_{j} \neq 0$ for some $j=0,1, \ldots, n$.

Thus, from the definition of $l$, we can see that $f \equiv t g$ where $t$ is a constant such that $t^{\kappa}=1, \kappa=G C D\left\{\lambda_{0}+\lambda, \lambda_{1}+\lambda, \ldots, \lambda_{n}+\lambda\right\}$.

Case 3. If $F(z) G(z) \equiv 1$. From Lemma 2.9 , we get that $f g=\mu$ for a constant $\mu$ such that $a_{n}^{2} \mu^{n+\lambda} \equiv 1$.

Thus, this completes the proof of Theorem 1.3.

Proof of Theorem 1.4 From the assumptions of Theorem 1.4, we have $E_{l}(1 ; F(z))=$ $E_{l}(1 ; G(z))$.

(I) $l \geq 3$. Since

$$
\begin{gathered}
\bar{N}\left(r, \frac{1}{F(z)-1}\right)+\bar{N}\left(r, \frac{1}{G(z)-1}\right)+\bar{N}_{(l+1}\left(r, \frac{1}{F(z)-1}\right) \\
+\bar{N}_{(l+1}\left(r, \frac{1}{G(z)-1}\right)-N_{11}\left(r, \frac{1}{F(z)-1}\right)
\end{gathered}
$$




$$
\begin{aligned}
& \leq \frac{1}{2} N\left(r, \frac{1}{F(z)-1}\right)+\frac{1}{2}\left(r, \frac{1}{G(z)-1}\right)+S(r, F)+S(r, G) \\
& \leq \frac{1}{2} T(r, F)+\frac{1}{2} T(r, G)+S(r, F)+S(r, G) .
\end{aligned}
$$

Case 1. Suppose that $F(z), G(z)$ satisfy Lemma 2.2(i). From (13), (14), and Lemma 2.6, we have

$$
(n+\lambda)[T(r, f)+T(r, g)] \leq 2\left(\Gamma_{0}+2 d\right)[T(r, f)+T(r, g)]+S_{1}(r, f)+S_{1}(r, g),
$$

that is,

$$
\left(n+\lambda-2 \Gamma_{0}-4 d\right)[T(r, f)+T(r, g)] \leq+S_{1}(r, f)+S_{1}(r, g) .
$$

Since $n>2 \Gamma_{0}+4 d-\lambda$ and $f, g$ are transcendental, a contradiction is obtained.

Case 2. If $F(z), G(z)$ satisfy Lemma 2.2(ii), that is,

$$
F=\frac{(b+1) G+(a-b-1)}{b G+(a-b)},
$$

where $a(\neq 0), b$ are two constants.

We now will consider three subcases as follows.

Subcase 2.1. $b \neq 0,-1$. If $a-b-1 \neq 0$, then by (20) we know

$$
\bar{N}\left(r, \frac{1}{G+\frac{a-b-1}{b+1}}\right)=\bar{N}\left(r, \frac{1}{F}\right) .
$$

Since $f, g$ are entire functions of zero order, by the Second Fundamental Theorem, we have

$$
\begin{aligned}
T(r, G) & \leq \bar{N}\left(r, \frac{1}{G}\right)+\bar{N}\left(r, \frac{1}{G+\frac{a-b-1}{b+1}}\right)+S(r, g) \leq \bar{N}\left(r, \frac{1}{G}\right)+\bar{N}\left(r, \frac{1}{F}\right)+S(r, g) \\
& \leq(m+d) T(r, g)+(m+d) T(r, f)+S_{1}(r, f)+S_{1}(r, g) .
\end{aligned}
$$

Then from Lemma 2.6, we have

$$
(n+\lambda-m-d) T(r, g) \leq(m+d) T(r, f)+S_{1}(r, f)+S_{1}(r, g) .
$$

Similarly, we have

$$
(n+\lambda-m-d) T(r, f) \leq(m+d) T(r, g)+S_{1}(r, f)+S_{1}(r, g) .
$$

From the definitions of $m$ and $\Gamma_{0}$, we have $m=m_{1}+m_{2}$. Since $n>2 \Gamma_{0}+4 d-\lambda$, we have $n+\lambda_{2} m-2 d>2 \Gamma_{0}+4 d-\lambda+\lambda-2 m-2 d=2 \Gamma_{0}+2 d-2 m>0$. From the above two inequalities, for any $\varepsilon\left(0<\varepsilon<2 \Gamma_{0}+2 d-2 m\right)$, we have

$$
(n+\lambda-2 m-2 d-\varepsilon)[T(r, f)+T(r, g)] \leq S_{1}(r, f)+S_{1}(r, g),
$$

which is a contradiction with $f, g$ are transcendental. 
If $a-b-1=0$, then by (20) we know $F=((b+1) G) /(b G+1)$. Since $f, g$ are entire functions, we see that $-\frac{1}{b}$ is a Picard's exceptional value of $G(z)$. By the Second Fundamental Theorem, we have

$$
T(r, G) \leq \bar{N}\left(r, \frac{1}{G}\right)+S(r, G) \leq(m+d) T(r, g)+S_{1}(r, g)
$$

Then, from Lemma 2.6 and $n>2 \Gamma_{0}+4 d-\lambda$, we know $T(r, g) \leq S_{1}(r, g)$, a contradiction.

Subcase 2.2. $b=-1$. Then (20) becomes $F=a /(a+1-G)$.

If $a+1 \neq 0$, then $a+1$ is a Picard exceptional value of $G$. Similar to the discussion as in Subcase 2.1, we can deduce a contradiction again.

If $a+1=0$, then $F G \equiv 1$, that is,

$$
P(f) \prod_{j=1}^{d} f\left(q_{j} z+c_{j}\right)^{s_{j}} P(g) \prod_{j=1}^{d} g\left(q_{j} z+c_{j}\right)^{s_{j}} \equiv 1 .
$$

Since $n>\max \left\{2 \Gamma_{0}+4 d-\lambda, \lambda\right\}$, by Lemma 2.9 , we see that $f g=\mu$ for a constant $\mu$ such that $a_{n}^{2} \mu^{n+\lambda} \equiv 1$.

Subcase 2.3. $b=0$. Then (20) becomes $F=(G+a-1) / a$.

If $a-1 \neq 0$, then $\bar{N}\left(r, \frac{1}{G+a-1}\right)=\bar{N}\left(r, \frac{1}{F}\right)$. Similar to the discussion as in Subcase 2.1, we can deduce a contradiction again.

If $a-1=0$, then $F \equiv G$, that is,

$$
P(f) \prod_{j=1}^{d} f\left(q_{j} z+c_{j}\right)^{s_{j}} \equiv P(g) \prod_{j=1}^{d} g\left(q_{j} z+c_{j}\right)^{s_{j}}
$$

Using the same argument as in the proof of Case 2 in Theorem 1.3, we can see that $f, g$ satisfy Theorem 1.3(ii).

(II) $l=2$. Since

$$
\begin{aligned}
\bar{N}\left(r, \frac{1}{F-1}\right)+\bar{N}\left(r, \frac{1}{G-1}\right)-N_{11}\left(r, \frac{1}{F-1}\right) \\
+\frac{1}{2} \bar{N}_{(l+1}\left(r, \frac{1}{F-1}\right)+\frac{1}{2} \bar{N}_{(l+1}\left(r, \frac{1}{G-1}\right) \\
\leq \frac{1}{2} N\left(r, \frac{1}{F-1}\right)+\frac{1}{2} N\left(r, \frac{1}{G-1}\right) \\
\leq \frac{1}{2} T(r, F)+\frac{1}{2} T(r, G)+S(r, F)+S(r, G), \\
\bar{N}_{(l+1}\left(r, \frac{1}{F-1}\right) \leq \frac{1}{2} N\left(r, \frac{F}{F^{\prime}}\right)=\frac{1}{2} N\left(r, \frac{F^{\prime}}{F}\right)+S(r, F) \\
\leq \frac{1}{2} \bar{N}\left(r, \frac{1}{F}\right)+S(r, F) \\
\leq \frac{m}{2} T(r, f)+\frac{d}{2} \bar{N}\left(r, \frac{1}{f}\right)+S_{1}(r, f),
\end{aligned}
$$


and

$$
\bar{N}_{(l+1}\left(r, \frac{1}{G-1}\right) \leq \frac{m}{2} T(r, g)+\frac{d}{2} \bar{N}\left(r, \frac{1}{g}\right)+S_{1}(r, g) .
$$

Case 1. If $F(z), G(z)$ satisfy Lemma 2.2(i), from the fact that $f(z), g(z)$ are transcendental entire functions and (21)-(22), we have

$$
\begin{aligned}
T(r, F(z))+T(r, G(z)) \leq & 2 N_{2}\left(r, \frac{1}{F}\right)+2 N_{2}\left(r, \frac{1}{G}\right)+m T(r, f)+m T(r, g) \\
& +d \bar{N}\left(r, \frac{1}{f}\right)+d \bar{N}\left(r, \frac{1}{g}\right)+S_{1}(r, f)+S_{1}(r, g) .
\end{aligned}
$$

From (13), (14), Lemma 2.6, and $\chi=\min \{\Theta(0, f), \Theta(0, g)\}$, for any $\varepsilon(0<\varepsilon<n+\lambda-m-$ $\left.2 \Gamma_{0}-5 d+d \chi\right)$, we have

$$
\left(n+\lambda-m-2 \Gamma_{0}-5 d+d \chi-\varepsilon\right)[T(r, f)+T(r, g)] \leq S_{1}(r, f)+S_{1}(r, g) .
$$

Since $n>2 \Gamma_{0}+5 d+m-\lambda-d \chi$ and $f, g$ are transcendental functions, we get a contradiction.

Case 2. If $F(z), G(z)$ satisfy Lemma 2.2(ii). Similar to the proof of Case 2 in (I), we get the conclusions of Theorem 1.4.

(III) $l=1$. Since

$$
\begin{gathered}
\bar{N}\left(r, \frac{1}{F-1}\right)+\bar{N}\left(r, \frac{1}{G-1}\right)-N_{11}\left(r, \frac{1}{F-1}\right) \\
\quad \leq \frac{1}{2} N\left(r, \frac{1}{F-1}\right)+\frac{1}{2} N\left(r, \frac{1}{G-1}\right) \\
\quad \leq \frac{1}{2} T(r, F)+\frac{1}{2} T(r, G)+S(r, F)+S(r, G),
\end{gathered}
$$

we have

$$
\begin{aligned}
\bar{N}_{(2}\left(r, \frac{1}{F}\right) & \leq N\left(r, \frac{F}{F^{\prime}}\right)=N\left(r, \frac{F^{\prime}}{F}\right)+S(r, f) \leq \bar{N}\left(r, \frac{1}{F}\right)+S(r, f) \\
& \leq m T(r, f)+d \bar{N}\left(r, \frac{1}{f}\right)+S_{1}(r, f),
\end{aligned}
$$

and

$$
\bar{N}_{(2}\left(r, \frac{1}{G}\right) \leq m T(r, g)+d \bar{N}\left(r, \frac{1}{g}\right)+S_{1}(r, g) .
$$

Case 1. If $F(z), G(z)$ satisfy Lemma 2.2(i), from $f, g$ are entire functions, (13), (14), (21), (22), (23), and (24), we have

$$
\begin{aligned}
T(r, F)+T(r, G) \leq & 2\left(\Gamma_{0}+2 d+m\right)[T(r, f)+T(r, g)]+2 d \bar{N}\left(r, \frac{1}{f}\right) \\
& +2 d \bar{N}\left(r, \frac{1}{g}\right)+S_{1}(r, f)+S_{1}(r, g) .
\end{aligned}
$$


From Lemma 2.6 and $\chi=\min \{\Theta(0, f), \Theta(0, g)\}$, for any $\varepsilon\left(0<\varepsilon<n+\lambda-2 \Gamma_{0}-6 d-2 m+\right.$ $2 d \chi)$, we have

$$
\left[n+\lambda-2 \Gamma_{0}-6 d-2 m+2 d \chi-\varepsilon\right][T(r, f)+T(r, g)] \leq S_{1}(r, f)+S_{1}(r, g) \text {. }
$$

Since $n>2 \Gamma_{0}+6 d+2 m-\lambda-2 d \chi$, from (27) and $f, g$ are transcendental, we get a contradiction.

Case 2. If $F(z), G(z)$ satisfy Lemma 2.2(ii). Similar to the proof of Case 2 in (I), we get the conclusions of Theorem 1.4.

(IV) $l=0$, that is, $F(z), G(z)$ share $1 I M$. From the definitions of $F(z), G(z)$, we have

$$
\begin{aligned}
\bar{N}_{L}\left(r, \frac{1}{F-1}\right) & \leq N\left(r, \frac{F}{F^{\prime}}\right)=N\left(r, \frac{F^{\prime}}{F}\right)+S(r, F) \leq \bar{N}\left(r, \frac{1}{F}\right)+S(r, F) \\
& \leq m T(r, f)+d \bar{N}\left(r, \frac{1}{f}\right)+S_{1}(r, f),
\end{aligned}
$$

similarly, we have

$$
\bar{N}_{L}\left(r, \frac{1}{G-1}\right) \leq m T(r, g)+d \bar{N}\left(r, \frac{1}{g}\right)+S_{1}(r, f) .
$$

Case 1. Suppose that $F(z), G(z)$ satisfy Lemma 2.3(i). From (28) and (29), we have

$$
\begin{aligned}
T(r, F(z))+T(r, G(z)) \leq & 2 N_{2}\left(r, \frac{1}{F}\right)+2 N_{2}\left(r, \frac{1}{G}\right)+3 m T(r, f)+3 m T(r, g) \\
& +3 d \bar{N}\left(r, \frac{1}{f}\right)+3 d \bar{N}\left(r, \frac{1}{g}\right)+S_{1}(r, f)+S_{1}(r, g) .
\end{aligned}
$$

From Lemma 2.6 and (13)-(14), for any $\varepsilon\left(0<\varepsilon<n+\lambda-2 \Gamma_{0}-7 d-3 m+3 d \chi\right)$, we get

$$
\left(n+\lambda-2 \Gamma_{0}-7 d-3 m+3 d \chi-\varepsilon\right)[T(r, f)+T(r, g)] \leq S_{1}(r, f)+S_{1}(r, g) .
$$

Since $n>2 \Gamma_{0}+7 d+3 m-\lambda-3 d \chi$, we get a contradiction.

Case 2. Suppose that $F(z), G(z)$ satisfy Lemma 2.3(ii). Similar to the proof of Case 2 in (I), we get the conclusions of Theorem 1.4 easily.

Thus, the proof of Theorem 1.4 is completed.

\section{Competing interests}

The authors declare that they have no competing interests.

\section{Authors' contributions}

HYX completed the main part of this article, XLW, HYX and TSZ corrected the main theorems. All authors read and approved the final manuscript.

\section{Author details}

${ }^{1}$ College of Science, University of Shanghai for Science and Technology, Shanghai, 200093, China. ${ }^{2}$ Department of Informatics and Engineering, Jingdezhen Ceramic Institute, Jingdezhen, Jiangxi 333403, China. 


\section{Acknowledgements}

The authors thank the referee for his/her valuable suggestions to improve the present article. This work was supported by the NSFC $(11301233,61202313)$, the Natural Science Foundation of Jiang-Xi Province in China (20132BAB211001), the Foundation of Education Department of Jiangxi (GJJ14644) of China, and the Humanities and Social Sciences of the Chinese Education Ministry (13YJA760064).

Received: 28 May 2014 Accepted: 9 September 2014 Published: 24 September 2014

\section{References}

1. Hayman, WK: Meromorphic Functions. Clarendon, Oxford (1964)

2. Yi, HX, Yang, CC: Uniqueness Theory of Meromorphic Functions. Kluwer Academic, Dordrecht (2003). Chinese original: Science Press, Beijing (1995)

3. Lahiri, I: Weighted sharing and uniqueness of meromorphic functions. Nagoya Math. J. 161, 193-206 (2001)

4. Lahiri, I: Weighted value sharing and uniqueness of meromorphic functions. Complex Var. Elliptic Equ. 46, 241-253 (2001)

5. Banerjee, A: Weighted sharing of a small function by a meromorphic function and its derivative. Comput. Math. Appl. 53, 1750-1761 (2007)

6. Barnett, DC, Halburd, RG, Korhonen, RJ, Morgan, W: Nevanlinna theory for the $q$-difference operator and meromorphic solutions of q-difference equations. Proc. R. Soc. Edinb., Sect. A, Math. 137, 457-474 (2007)

7. Chiang, YM, Feng, SJ: On the Nevanlinna characteristic of $f(z+\eta)$ and difference equations in the complex plane. Ramanujan J. 16, 105-129 (2008)

8. Halburd, RG, Korhonen, RJ: Difference analogue of the lemma on the logarithmic derivative with applications to difference equations. J. Math. Anal. Appl. 314, 477-487 (2006)

9. Halburd, RG, Korhonen, RJ: Finite-order meromorphic solutions and the discrete Painleve equations. Proc. Lond. Math. Soc. 94, 443-474 (2007)

10. Halburd, RG, Korhonen, RJ: Nevanlinna theory for the difference operator. Ann. Acad. Sci. Fenn., Math. 31, 463-478 (2006)

11. $\mathrm{Xu}, \mathrm{HY}$ : On the value distribution and uniqueness of difference polynomials of meromorphic functions. Adv. Differ. Equ. 2013, Article ID 90 (2013)

12. $\mathrm{Xu}, \mathrm{HY}$, Liu, BX, Tang, KZ: Some properties of meromorphic solutions of systems of complex $q$-shift difference equations. Abstr. Appl. Anal. 2013, Article ID 680956 (2013)

13. $\mathrm{Xu}, \mathrm{HY}, \mathrm{Tu}, \mathrm{J}$, Zheng, XM: Some properties of solutions of complex $q$-shift difference equations. Ann. Pol. Math. 108(3) 289-304 (2013)

14. Liu, K, Liu, XL, Cao, TB: Value distributions and uniqueness of difference polynomials. Adv. Differ. Equ. 2011, Article ID $234215(2011)$

15. Chen, ZX, Huang, ZB, Zheng, XM: On properties of difference polynomials. Acta Math. Sci. 31, 627-633 (2011)

16. Luo, XD, Lin, WC: Value sharing results for shifts of meromorphic functions. J. Math. Anal. Appl. 377, 441-449 (2011)

17. Zhang, JL, Korhonen, RJ: On the Nevanlinna characteristic of $f(q z)$ and its applications. J. Math. Anal. Appl. 369, 537-544 (2010)

18. Liu, K, Qi, XG: Meromorphic solutions of q-shift difference equations. Ann. Pol. Math. 101, 215-225 (2011)

19. Liu, K, Cao, HZ, Cao, TB: Entire solutions of Fermat type differential difference equations. Arch. Math. 99, 147-155 (2012)

20. Liu, K, Yang, LZ: On entire solutions of some differential-difference equations. Comput. Methods Funct. Theory 13 433-447 (2013)

21. Chen, ZX: On value distribution of difference polynomials of meromorphic functions. Abstr. Appl. Anal. 2011, Article ID 239853 (2011)

22. Heittokangas, J, Korhonen, RJ, Laine, I, Rieppo, J, Zhang, JL: Value sharing results for shifts of meromorphic functions, and sufficient conditions for periodicity. J. Math. Anal. Appl. 355, 352-363 (2009)

23. Laine, I, Yang, CC: Value distribution of difference polynomials. Proc. Jpn. Acad., Ser. A, Math. Sci. 83, 148-151 (2007)

24. Fang, CY, Fang, ML: Uniqueness of meromorphic functions and differential polynomials. Comput. Math. Appl. 44, 607-617 (2002)

25. Zhang, JL, Yang, LZ: Some results related to a conjecture of R. Brück. J. Inequal. Pure Appl. Math. 8, 18 (2007)

doi:10.1186/1687-1847-2014-249

Cite this article as: Wang et al.: Properties of $q$-shift difference-differential polynomials of meromorphic functions. Advances in Difference Equations 2014 2014:249. 